\title{
VEHICLE STEERING CONTROL BASED ON ESTIMATION OF EQUIVALENT INPUT DISTURBANCE
}

\author{
Jin-Hua She ${ }^{*}$ Xin Xin ${ }^{* *}$ Yasuhiro Ohyama* \\ Min Wu ${ }^{* * *}$ Hiroyuki Kobayashi* \\ * School of Bionics, Tokyo University of Technology \\ 1404-1 Katakura, Hachioji, Tokyo, 192-0982 Japan \\ E-mail: $\{$ she, ohyama, yagshi\}@bs.teu.ac.jp \\ ** Faculty of Computer Science \& Computing \\ Okayama Prefectural University \\ 111 Kuboki, Soja, Okayama, 719-1197 Japan \\ E-mail: xxin@c.oka-pu.ac.jp \\ *** School of Information Science $\&$ Engineering \\ Central South University \\ Changsha, Hunan, 410083 China \\ E-mail: min@mail.csu.edu.cn
}

\begin{abstract}
This paper describes a simple but effective approach to vehicular steering control based on the estimation of an equivalent input disturbance. A control system configuration employing the disturbance estimate is presented, and some simulation results are provided to demonstrate the validity of the method. Copyright $\left.{ }^{(}\right) 2005$ IFAC
\end{abstract}

Keywords: vehicle dynamics, vehicle control, vehicle steering, disturbance estimation, disturbance rejection, $H_{\infty}$ control, linear matrix inequality (LMI).

\section{INTRODUCTION}

Automatic steering control is a key element of intelligent transportation systems, and has been intensively investigated, e.g., (O'Brien et al., 1996; Byrne et al., 1998; Hatipoglu et al., 2003; Kim et al., 2001; Peng and Tomizuka, 1993; Lin et al., 2000; Güvenç et al., 2001; Yamamoto et al., 1999). It involves two techniques: lane keeping, for which the steering control system must track the center of the current lane; and lane changing, for which it must steer so as to track a reference input for a given lateral motion.
Since disturbances caused by wind and road conditions seriously affect the control results, disturbance rejection performance is a key figure of merit of a steering control system; and many researchers have studied disturbance rejection in steering control. For example, (Lin et al., 2000) used a steady-state Kalman filter to estimate the lateral velocity of the vehicle and the magnitudes of external disturbances acting on the vehicle; (Güvenç et al., 2001) applied the disturbance observer approach to suppress external disturbances; and (Yamamoto et al., 1999) proposed a disturbance estimation algorithm. However, each method has drawbacks: (Lin et al., 2000) con- 


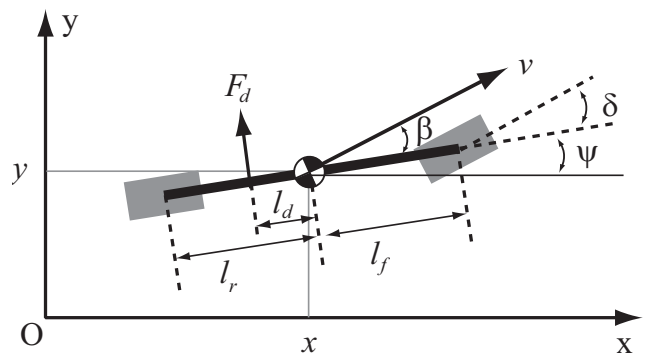

Fig. 1. Bicycle model of vehicle (top view).

sidered only step-type disturbances; in (Güvenç et al., 2001), it is difficult to tune the filter parameters because the filter has to be designed to guarantee both the causality of the estimator and the stability of the whole control system; and (Yamamoto et al., 1999) requires the differentiation of measured outputs.

This paper addresses the steering control problem for a straight road. A new estimation method called an equivalent input disturbance estimator (She et al., 2004) is employed to estimate an equivalent disturbance on the control input channel to reject disturbances caused by wind, yaw disturbance torque, etc., and thus provide good steering performance. The approach proposed in this paper has three advantages: First, no a priori information about disturbances is required. The only restriction is that the output produced by disturbances must be bounded and smooth; but this condition is generally satisfied. Second, no differentiation of measured outputs is needed. Third, the design of the control system is very simple; that is, the control system configuration can be viewed as a conventional servo system enhanced by the plugging-in of a disturbance estimate. Simulation results have demonstrated the validity of this method.

\section{MODEL OF VEHICLE}

This paper considers a vehicle with front-wheel steering. It is assumed that there are no sudden accelerations or decelerations, and that the front wheels are only turned through a small angle. So, the velocity is constant, and the rolling motion can be ignored. Thus, the vehicle can be thought of as traveling on a flat surface, affected only by lateral and yawing motions. A further assumption is that the sideslip angle and the yaw rate are very small.

Since a complicated model is not necessary to simulate the lateral motion of highway vehicles, as pointed out in (O'Brien et al., 1995), this study employed the simple 2-degree-of-freedom model (often called the "bicycle model" (Kumamoto et al., 1998)) shown in Fig. 1. The parameters are: $y$ : y-position of center of gravity (CG) of vehicle $[\mathrm{m}]$.

$\psi$ : yaw angle with respect to $\mathrm{x}$-axis [rad].

$\delta$ : angle of front wheel [rad].

$\beta$ : sideslip angle [rad].

$v$ : longitudinal velocity $[\mathrm{m} / \mathrm{s}]$.

$m$ : total mass of vehicle $[\mathrm{kg}]$.

$I_{z}$ : yaw moment of inertia $\left[\mathrm{kg} \cdot \mathrm{m}^{2}\right]$.

$l_{f}\left(l_{r}\right)$ : distance from CG to front (rear) axle [m].

$l_{d}$ : distance from CG to position at which $F_{d}$ has an effect $[\mathrm{m}]$.

$C_{f}\left(C_{r}\right)$ : cornering stiffness of front (rear) tires $[\mathrm{N} / \mathrm{rad}]$.

$F_{d}$ : equivalent lateral disturbance force $[\mathrm{N}]$.

$\tau_{d}$ : equivalent yaw disturbance torque $[\mathrm{Nm}]$.

The dynamic equations of the lateral motion are

$$
\left\{\begin{aligned}
m \frac{d^{2} y}{d t^{2}}= & -\frac{C_{f}+C_{r}}{v} \frac{d y}{d t}+\left(C_{f}+C_{r}\right) \psi \\
& -\frac{C_{f} l_{f}-C_{r} l_{r}}{v} \frac{d \psi}{d t}+C_{f} \delta+F_{d}, \\
I_{z} \frac{d^{2} \psi}{d t^{2}}= & -\frac{C_{f} l_{f}-C_{r} l_{r}}{v} \frac{d y}{d t}+\left(C_{f} l_{f}-C_{r} l_{r}\right) \psi \\
& -\frac{C_{f} l_{f}^{2}+C_{r} l_{r}^{2}}{v} \frac{d \psi}{d t}+C_{f} l_{f} \delta+\tau_{d} .
\end{aligned}\right.
$$

The state-space expression is

$$
\left\{\begin{array}{l}
\frac{d \xi}{d t}=A \xi+B \delta+B_{d} d \\
y=C \xi
\end{array}\right.
$$

where

$$
\begin{aligned}
& \xi=\left[\begin{array}{llll}
y & \frac{d y}{d t} & \psi & \frac{d \psi}{d t}
\end{array}\right]^{T}, \\
& d=\left[\begin{array}{llll}
F_{d} & \tau_{d}
\end{array}\right]^{T}, \\
& A=\left[\begin{array}{cccc}
0 & 1 & 0 & 0 \\
0 & \frac{a_{1}}{v} & -a_{1} & \frac{a_{2}}{v} \\
0 & 0 & 0 & 1 \\
0 & \frac{a_{3}}{v} & -a_{3} & \frac{a_{4}}{v}
\end{array}\right], B=\left[\begin{array}{c}
0 \\
b_{1} \\
0 \\
b_{2}
\end{array}\right], \\
& B_{d}=\left[\begin{array}{cc}
0 & 0 \\
b_{d 1} & 0 \\
0 & 0 \\
0 & b_{d 2}
\end{array}\right], C=\left[\begin{array}{c}
1 \\
0 \\
0 \\
0
\end{array}\right], \\
& a_{1}=-\frac{C_{f}+C_{r}}{m}, a_{2}=-\frac{C_{f} l_{f}-C_{r} l_{r}}{m}, \\
& a_{3}=-\frac{C_{f} l_{f}-C_{r} l_{r}}{I_{z}}, a_{4}=-\frac{C_{f} l_{f}^{2}+C_{r} l_{r}^{2}}{I_{z}}, \\
& b_{1}=\frac{C_{f}}{m}, b_{2}=\frac{C_{f} l_{f}}{I_{z}}, b_{d 1}=\frac{1}{m}, b_{d 2}=\frac{1}{I_{z}} .
\end{aligned}
$$

Note that the relationship between $F_{d}$ and $\tau_{d}$ is $\tau_{d}=F_{d} l_{d}$. For this model, $(A, B)$ is controllable and $(C, A)$ is observable. The next section concerns the design of a steering control system based on $(2)$.

\section{DESIGN OF CONTROL SYSTEM}

Since the steering angle, $\delta$, is the only control input, it is more reasonable to estimate an equivalent disturbance on the control input channel than 


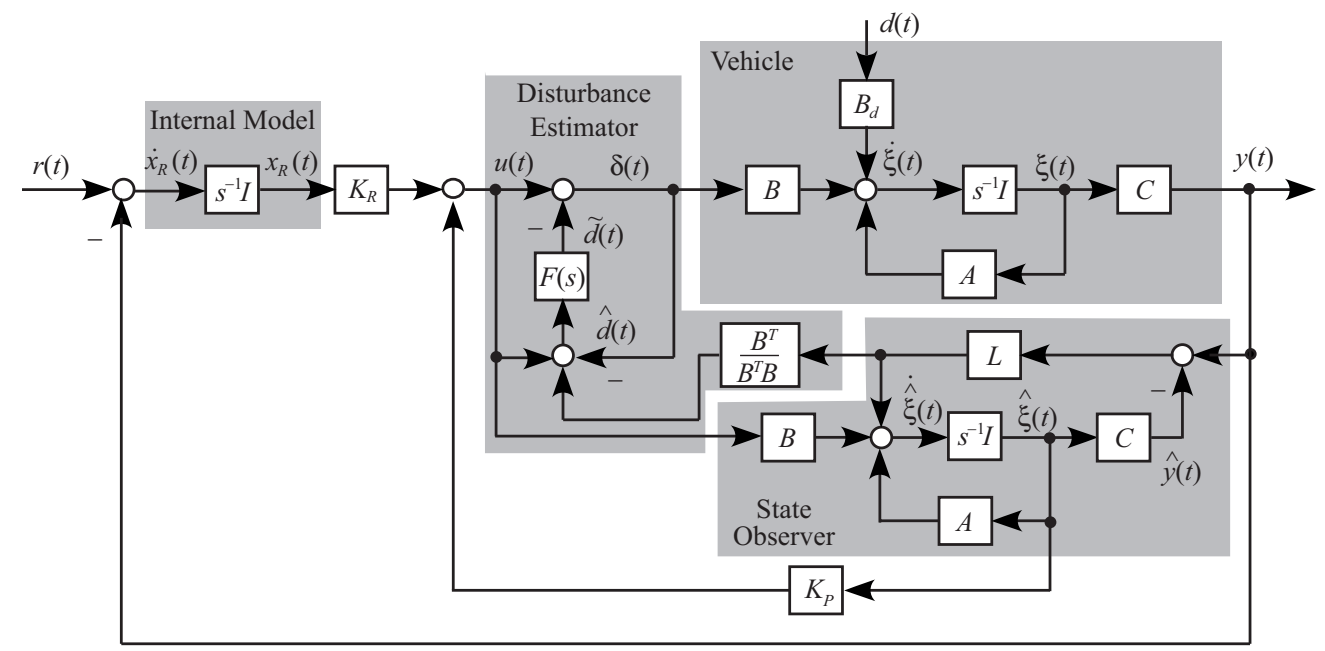

Fig. 2. Configuration of steering control system.

to estimate the disturbances themselves. Since an equivalent input disturbance exists ((She et $a l ., 2004)$ ), we focused on its estimation and constructed the steering control system in Fig. 2. This configuration can be viewed as a conventional servo system enhanced by the plugging-in of a disturbance estimate. Since the lane-change command can be characterized as a step signal, a step-type servo system was selected.

For a conventional servo system, the closed-loop eigenvalues are those of the full-state feedback plus those of the estimator (the Separation Theorem) (Anderson and Moore, 1989). A simple calculation shows that the separation property also holds for our system configuration, i.e., the closed-loop eigenvalues are those of the full-state feedback plus those of the estimator and low-pass filter. So, the rest of this paper focuses on the design of the estimator and the low-pass filter.

\subsection{Estimation and rejection of disturbances}

Since the state is estimated by a full-order state observer of the vehicle,

$$
\frac{d \hat{\xi}}{d t}=A \hat{\xi}+B u+L C(\xi-\hat{\xi})
$$

is true. On the other hand, since an estimate is obtained for an equivalent input disturbance, the estimated state of the plant might be different from the actual state resulting from the effects of the disturbance. We can take the state of the plant with an equivalent input disturbance to be $\hat{\xi}(t)$, which is exactly the state of the observer, and consider the difference between the output of the real plant and that of the plant with an equivalent input disturbance arising from the difference between the exact value and the estimate of the equivalent input disturbance. Thus,

$$
\frac{d \hat{\xi}}{d t}=A \hat{\xi}+B(\delta+\hat{d})
$$

where $\hat{d}$ is the estimate of the equivalent input disturbance. Controllability requires $B^{T} B \neq 0$. Thus, (4) and (3) yield

$$
\hat{d}=\frac{B^{T}}{B^{T} B} L C(\xi-\hat{\xi})+u-\delta
$$

Then, the disturbance estimate, $\tilde{d}(t)$, is obtained by filtering out $\hat{d}(t)$ with a low-pass filter, $F(s)$, as shown in Fig. 2. Combining $\tilde{d}(t)$ with the original control law yields

$$
\delta=u-\tilde{d}
$$

This modified control law improves the disturbance rejection performance.

This method has the following features:

1) The configuration of the control system is very simple.

2) The disturbance rejection performance can be tuned by adjusting $F(s)$ and the observer gain, $L$.

3) The stability of the closed-loop system can be divided into two parts: the feedback gains $K_{P}$ and $K_{R}$, and $L$ and $F(s)$.

The only difference between this system and a conventional servo system is the incorporation of the disturbance estimate; and the only design parameters related to that estimate are the low-pass filter and observer, which are discussed below.

\subsection{Design of observer}

$F(s)$ and $L$ should be designed so that they do not destroy the stability of the control system. Focusing on the stability issue, we let $r(t)=0$ and $d(t)=0$. We also introduce a new variable $\Delta \xi(t)=\hat{\xi}(t)-\xi(t)$. Now, the vehicle is described by

$$
\frac{d \xi}{d t}=A \xi+B \delta
$$

(3), (6) and (7) yield 


$$
\frac{d \Delta \xi}{d t}=(A-L C) \Delta \xi+B \tilde{d} .
$$

On the other hand, (5) is equivalent to

$$
\hat{d}=-\frac{B^{T}}{B^{T} B} L C \Delta \xi+\tilde{d} .
$$

So, the transfer function from $\tilde{d}(t)$ to $\hat{d}(t)$ is

$$
G(s)=1-\frac{B^{T}}{B^{T} B} L C[s I-(A-L C)]^{-1} B .
$$

The Small-Gain Theorem tells us that, when the disturbance estimate is incorporated into the control system, the stability is guaranteed if

$$
\|G F\|_{\infty}<1 \text {. }
$$

If we choose the angular frequency band for disturbance rejection to be

$$
\Omega_{r}=\left\{\omega: \omega \leq \omega_{r}\right\},
$$

then, to reject disturbances, it is best to choose $|F(j \omega)| \approx 1$ (and $|F(j \omega)| \leq 1$ ) in the range $\Omega_{r}$. It is clear from Condition (11) and Fig. 2 that we have to select $G(s)$ such that $|G(j \omega)|<1$ holds in $\Omega_{r}$. In what follows, we shall show that the design of $L$ can be transformed to an $H_{\infty}$ static output feedback synthesis problem.

Letting the state space representations of $F(s)$ and $G(s)$ be

$$
\begin{aligned}
& F(s):\left\{\begin{array}{l}
\frac{d x_{f}}{d t}=A_{f} x_{f}+B_{f} w, \\
y_{f}=C_{f} x_{f},
\end{array}\right. \\
& G(s):\left\{\begin{array}{l}
\frac{d x_{L}}{d t}=A x_{L}+B y_{f}-u_{L}, \\
z=y_{f}-\frac{B^{T}}{B^{T} B} u_{L}, \\
y_{L}=C x_{L}, \\
u_{L}=L y_{L},
\end{array}\right.
\end{aligned}
$$

then we can construct the following general plant $G_{g}(s)=G(s) F(s)$ :

$$
\left\{\begin{array}{l}
{\left[\begin{array}{c}
\frac{d x_{f}}{d t} \\
\frac{d x_{L}}{d t}
\end{array}\right]=\left[\begin{array}{cc}
A_{f} & 0 \\
B C_{f} & A
\end{array}\right]\left[\begin{array}{c}
x_{f} \\
x_{L}
\end{array}\right]+\left[\begin{array}{c}
B_{f} \\
0
\end{array}\right] w+\left[\begin{array}{c}
0 \\
-I
\end{array}\right] u_{L},} \\
z=\left[\begin{array}{ll}
C_{f} & 0
\end{array}\right]\left[\begin{array}{l}
x_{f} \\
x_{L}
\end{array}\right]-\frac{B^{T}}{B^{T} B} u_{L}, \\
y_{L}=\left[\begin{array}{ll}
0 & C
\end{array}\right]\left[\begin{array}{l}
x_{f} \\
x_{L}
\end{array}\right] .
\end{array}\right.
$$

The design problem now becomes to design a control law,

$$
u_{L}=L y_{L},
$$

such that

$$
\left\|G_{g}\right\|_{\infty}<1
$$

Note that the $H_{\infty}$ static output feedback synthesis problem is not convex. The cone complementarity linearization algorithm (Ghaoui et al., 1997) can be employed to tackle this problem. In order to achieve good control performance, $L$ is designed such that all the poles of $G_{g}(s)$ lie in the half plane of $\operatorname{Re}(s) \leq-\alpha$ ( $\alpha$ : a positive real number). The problem can be solved by combining the complementarity linearization algorithm with the LMIbased $\alpha$-stable design algorithm for $H_{\infty}$ control (Gahinet and Apkarian, 1994).
Based on the above, we can now give a design algorithm for the steering control system.

\section{Design algorithm:}

Step 1. Design $K_{P}$ and $K_{R}$ for a conventional step-type servo system by an existing method (e.g., the optimal control method).

Step 2. Choose $\Omega_{r}$ and a corresponding $F(s)$.

Step 3. Design $L$ by combining the cone complementarity linearization algorithm (Ghaoui et al., 1997) with the LMI-based $\alpha$-stable design algorithm for $H_{\infty}$ control (Gahinet and Apkarian, 1994).

\section{SIMULATIONS}

Simulations were carried out for a straight road using the vehicle parameters in (Kumamoto et al., 1998), which are listed in Table 1. First, we chose

$$
Q=\operatorname{diag}\{100,1,1,1,100\}, R=10000,
$$

and obtained $K_{P}$ and $K_{R}$ by optimizing the following performance index:

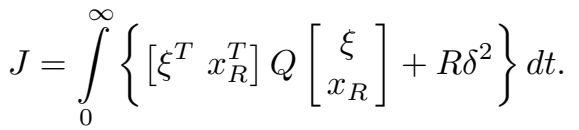

The resulting $K_{P}$ and $K_{R}$ are

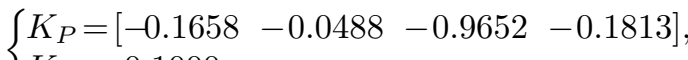
$\left\{K_{R}=0.1000\right.$.

Then, we selected $\Omega_{r}$ by setting $\omega_{r}$ to $30 \mathrm{rad} / \mathrm{s}$ and $F(s)$ to

$$
F(s)=\frac{1}{T s+1}, T=0.0333 \mathrm{~s} .
$$

Finally, using the design algorithm proposed in the last section to solve the $H_{\infty}$ synthesis problem (13)-(15) yielded the observer gain $(\alpha=1.82)$

$$
L=\left[\begin{array}{llll}
168.94 & 751.97 & 153.87 & 261.27
\end{array}\right]^{T} .
$$

The resulting transfer function is

$$
\begin{aligned}
& G(s)=\frac{s^{4}+n_{3} s^{3}+n_{2} s^{2}+n_{1} s+n_{0}}{s^{4}+d_{3} s^{3}+d_{2} s^{2}+d_{1} s+d_{0}}, \\
& d_{3}=174.68, d_{2}=1739.4, d_{1}=17494, \\
& d_{0}=58592, n_{3}=174.68, n_{2}=1071.2, \\
& n_{1}=15467, n_{0}=19614 .
\end{aligned}
$$

A Bode plot of $G(s) F(s)$ is shown in Fig. 3 . Clearly, it satisfies (11).

\subsection{Simulation results for lane-changing control}

Figure 4 shows the ideal lateral response (dash-dot line) and steering angle of a vehicle with nominal

Table 1. Vehicle parameters and conditions used in the simulations.

\begin{tabular}{cccc}
\hline$m[\mathrm{~kg}]$ & $I_{z}\left[\mathrm{kgm}^{2}\right]$ & $v[\mathrm{~m} / \mathrm{s}]$ & $l_{d}[\mathrm{~m}]$ \\
\hline 1500 & 3000 & 25 & -1.2 \\
\hline \hline$C_{f}[\mathrm{~N} / \mathrm{rad}]$ & $C_{r}[\mathrm{~N} / \mathrm{rad}]$ & $l_{f}[\mathrm{~m}]$ & $l_{r}[\mathrm{~m}]$ \\
\hline 50000 & 70000 & 1.2 & 1.3 \\
\hline
\end{tabular}




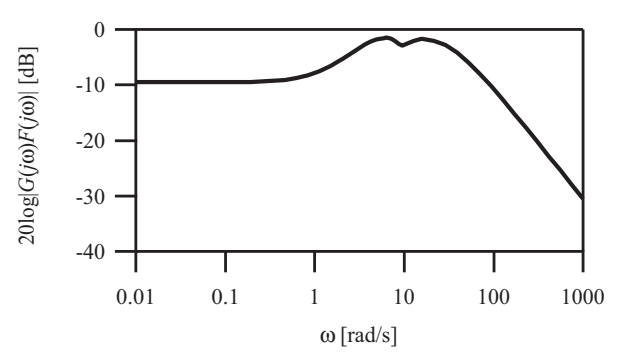

Fig. 3. Bode plot of $G(s) F(s)$.
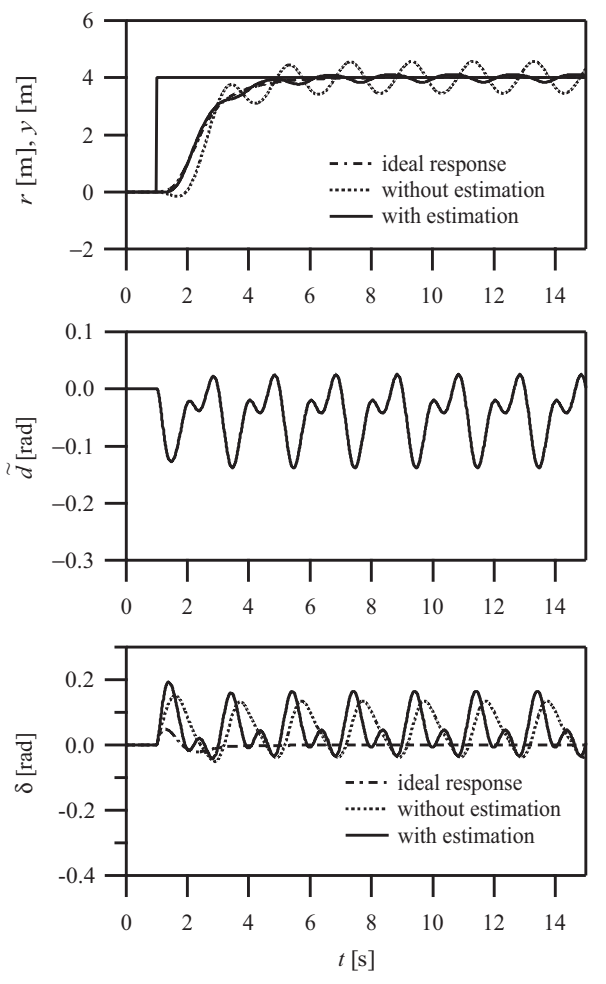

Fig. 4. Simulation results for lane-changing control of vehicle with nominal parameters.

parameters under zero initial conditions when the reference input for the lane change is

$$
r(t)=4 \times 1(t-1) \mathrm{m} .
$$

Next, the following disturbances (Fig. 5) were added:

$$
\left\{\begin{array}{c}
F_{d}=-2000 \times[1+\sin \pi(t-1) \\
\quad+0.5 \sin 2 \pi(t-1)+0.5 \sin 20 \pi(t-1)] \mathrm{N} \\
\tau_{d}=2400 \times[1+\sin \pi(t-1) \\
\quad+0.5 \sin 2 \pi(t-1)+0.5 \sin 20 \pi(t-1)] \mathrm{Nm} .
\end{array}\right.
$$

Figure 4 also shows the response without disturbance estimation (dotted line) and with the disturbance estimate (solid line) obtained by our method. Incorporating the disturbance estimate dramatically reduced the peak-to-peak tracking error from $1.1097 \mathrm{~m}$ to $0.2577 \mathrm{~m}$.

Stability tests showed that the designed control system is stable for parameters in the following ranges:

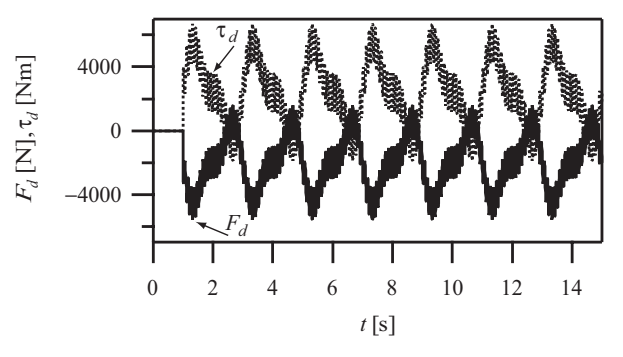

Fig. 5. Disturbances.
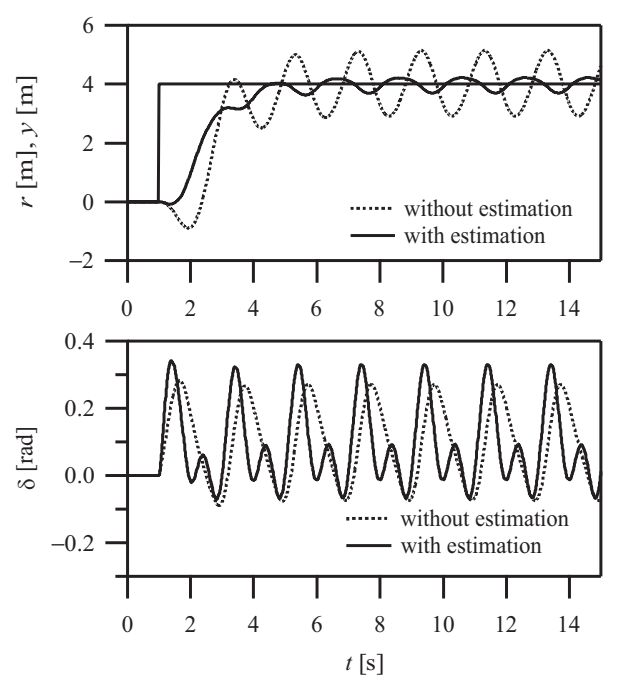

Fig. 6. Simulation results for lane-changing control of a vehicle with the parameters in (25).

$$
\left\{\begin{array}{l}
m=1500 \times(100 \pm 50) \% \mathrm{~kg}, \\
I_{z}=3000 \times(100 \pm 50) \% \mathrm{kgm}^{2} \\
C_{f}=50000 \times(100 \pm 50) \% \mathrm{~N} / \mathrm{rad}, \\
C_{r}=70000 \times(100 \pm 50) \% \mathrm{~N} / \mathrm{rad} .
\end{array}\right.
$$

Figure 6 shows the results for the combination of parameters

$$
\begin{cases}m=750 \mathrm{~kg}, & I_{z}=1500 \mathrm{kgm}^{2}, \\ C_{f}=25000 \mathrm{~N} / \mathrm{rad}, & C_{r}=35000 \mathrm{~N} / \mathrm{rad},\end{cases}
$$

and the disturbances in (23) under zero initial conditions. Clearly, even though the parameters were different from the nominal values, the disturbances were suppressed.

\subsection{Simulation results for lane-keeping control}

The simulations also demonstrated that incorporating the disturbance estimate markedly reduces the tracking error for lane-keeping control. As an example, Fig. 7 shows some results for a straight road and a vehicle with the parameters in (25) under zero initial conditions when the disturbances in (23) were added.

\section{CONCLUSIONS}

This paper presents a new solution to the steering control problem for a vehicle with front-wheel 

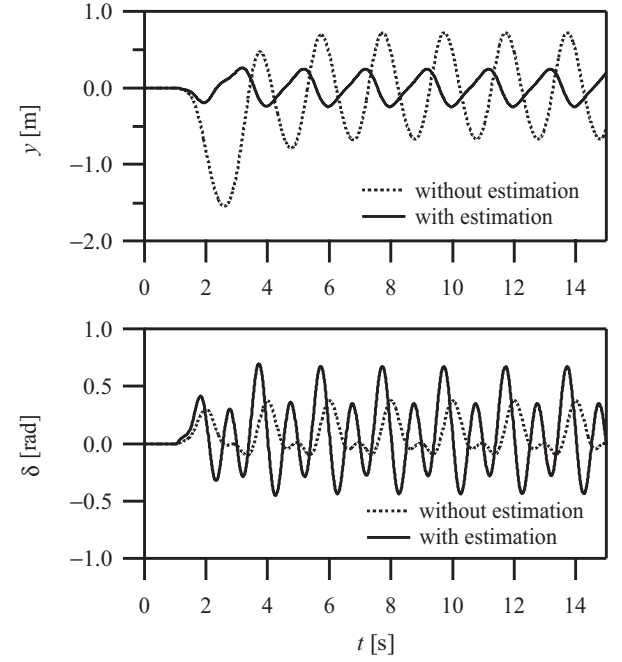

Fig. 7. Simulation results for lane-keeping control of a vehicle with the parameters in (25).

steering. First, the concept of equivalent input disturbance was explained. Next, a method of estimating an equivalent input disturbance was described. Then, a steering control configuration that employs the disturbance estimate was presented. Unlike existing methods, this one is very simple. Furthermore, the design of the parameters related to the disturbance estimation can be separated from that for a conventional servo controller, and an algorithm was presented for their design. Simulation results demonstrated the validity of the method.

This study only considered steering control for a straight road; but a general formulation must take the radius of curvature (ROC) of the road into account. The problem can then be divided into two cases based on whether or not preview information on the road ROC is available. If it is, a steering control system can be designed using a feedforward control scheme (Peng and Tomizuka, 1993); but if it is not, the control problem can be formulated as a disturbance rejection problem (O'Brien et al., 1996). So, even when the road ROC cannot be used, our method can still be employed to estimate the equivalent disturbance due to the road ROC; and thus satisfactory control can be achieved.

\section{REFERENCES}

Anderson, B.D.O. and J.B. Moore (1989). Optimal Control -Linear Quadratic Methods-. Prentice-Hall, Inc. Englewood Cliffs, NJ.

Byrne, R.H., C.T. Abdallah and P. Dorato (1998). Experimental results in robust lateral control of highway vehicles. IEEE Control Systems Magazine 18, 70-76.
Ghaoui, El L., F. Oustry and M. AitRami (1997). A cone complementary linearization algorithm for static output-feedback and related problems. IEEE Trans. on Automatic Control, 42, 1171-1176.

Gahinet, P. and P. Apkarian (1994). A linear matrix inequality approach to $H_{\infty}$ control. Int. J. Robust and Nonlinear Control, 4, 421448.

Güvenç, B.A., T. Bünte, D. Odenthal and L. Günenç (2001). Robust two degree of freedom vehicle steering controller design. Proc. of the 2001 American Control Conference, $13-18$.

Hatipoglu, C., Ü. Özguner and K. Redmill (2003). Automated lane change controller design. IEEE Trans. on Intelligent Transportation Systems 4, 13-22.

Kim, C.S., J.I. Bae, S.P. Hong, M.H. Lee and F. Harashima (2001). $H_{\infty}$ steering control for the unmanned vehicle system. Proc. of the 27th Annual Conference of the IEEE Industrial Electronics Society (IECON'01), 21392143.

Kumamoto, H., K I. Sakamoto, Tenmoku and H. Shimoura (1998). Vehicle steering control by reduced-dimension sliding mode theory. Trans. of SICE (in Japanese) 34, 393-399.

Lin, C.-F., A.G. Ulsoy and D.J. LeBlanc (2000). Vehicle dynamics and external disturbance estimation for vehicle path prediction. IEEE Trans. on Control Systems Technology 8, 508-518.

O'Brien, R.T., P.A. Iglesias and T.J. Urban (1996). Vehicle lateral control for automated highway systems. IEEE Trans. on Control Systems Technology 4, 266-273.

O'Brien, R.T., T.J. Urban and P.A. Iglesias (1995). Lane change maneuver via modern steering control methods. Proc. ITS America Conference 1995, 1-9.

Peng, H. and M. Tomizuka (1993). Preview control for vehicle lateral guidance in highway automation. Trans. ASME J. Dyn. Syst. Measurement, Contr. 115, 679-686.

She, J.-H., H. Kobayashi, Y. Ohyama and X. Xin (2004). Disturbance estimation and rejection -An equivalent input disturbance estimator approach-. Proc. 43rd IEEE Conf. Decision Control, 1736-1741.

Yamamoto, M., Y. Kagawa and A. Okuno (1999). Robust control for automated lane keeping against lateral disturbance. Proc. of 1999 IEEE/IEEJ/JSAI International Conference on Intelligent Transportation Systems, 240245. 